To the Editors:

\title{
Could the latex of Semecarpus anacardium (Kiri Badulla) cause nephrotic syndrome?
}

Ceylon Medical Journal 2012; 57: 92-93

\section{Introduction}

Minimal change disease (MCD) accounts for $10-15 \%$ of primary nephrotic syndrome cases in adults [1]. Most cases are idiopathic. However, approximately $10 \%$ may have an identifiable cause. Secondary causes may include drugs, toxins, infections and tumours [2]. Non-steroidal anti-inflammatory drugs (NSAIDs) mercury, lithium and bee stings are some established causes of MCD. Although some plant products are known to cause nephrotoxicity, external contact of plant latex has not been reported as a nephrotoxin. We are reporting a possible case of an unusual cause of nephrotic syndrome following skin contact of latex of Semecarpus anacardium (SA).

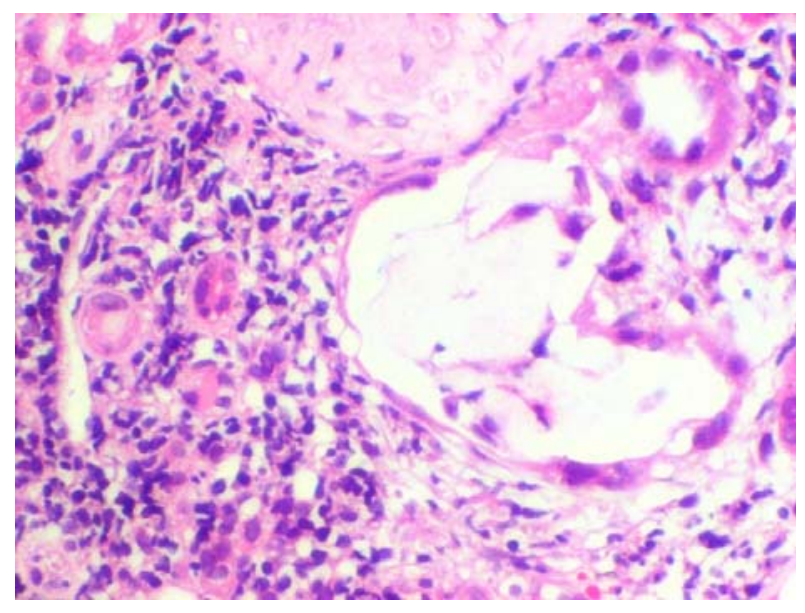

Figure. Renal histology showing protein casts in a tubule and interstitial lymphocytic infiltration.

\section{Case report}

A 27-year old previously healthy male presented with features of nephrotic syndrome following skin contact with latex from the bark of SA. Immediately following the contact, he developed generalised urticarial pruritic rash which resolved spontaneously over a period of 24 hours. From the 3rd day of exposure, he developed generalised body swelling with reduced urine output. There was no history suggestive of any infections, drug ingestion or insect bites. Examination revealed generalised oedema but cardiac, neurological and respiratory systems were clinically normal. Investigations confirmed heavy proteinuria (more than $3 \mathrm{~g}$ a day), hypoalbunaemia and oedema suggestive of nephrotic syndrome. Urinary sediments revealed 10-15 pus cells without red cells or red cell casts. He had an elevated serum creatinine level of $156.2 \mu \mathrm{ml} / \mathrm{l}$. Anti nuclear antibody was negative. Ultrasound scan of kidneys were normal and renal histology showed an acute interstitial nephritis with negative immunofluorescence (Figure). He responded well to high doses of oral prednisolone and was free of proteinuria at three months.

\section{Discussion}

SA is a plant distributed mainly in India and some other parts of subtropical regions [3]. Many parts of this plant have been used in indigenous medicine since ancient times [3]. A variety of nut extract preparations from this source have been effectively used against many diseases like arthritis, tumours and infections [4]. Chemical and phytochemical analyses of SA nut have revealed the presence of biflavonoids, phenolic compounds, biflavones, minerals, vitamins and amino acids [4]. Ethyl acetate extract of SA, contains 3,4,2',4'-tetrahydroxychalcone (butein) and 7,3',4'-trihydroxy (flavone), which has COX-1 and to a certain extent COX-2 inhibitory activity.

Here we report a case of nephrotic syndrome following skin contact with latex of SA. Light microscopy revealed normal glomeruli and interstitial nephritis. Heavy proteinuria cannot only be explained by light microscopic findings. Therefore we suspected co-existing minimal change disease which might have caused heavy proteinuria and hypoprotenaemia. Unfortunately electron microscopy had not been performed due to unavailability. The chemical components of the plant that have COX inhibitory activities might be responsible for the nephrotic syndrome and interstitial nephritis. Similar presentation is seen in NSAID induced acute kidney injury in which an acute interstitial nephritis with an interstitial infiltrate of T lymphocytes and the nephrotic syndrome due to minimal change disease [5]. So we suggest renal injury similar to NSAIDs exposure could occur following exposure to SA which has COX-1 and COX-2 inhibitory activity.

\section{References}

1. Cameron JS. The nephrotic syndrome and its complications. American Journal of Kidney Diseases 1987; 10: 157-71. 
2. Almansori M, Kovithavongs T, Qarni MU. Cyclooxygenase2 inhibitor-associated minimal-change disease. Clinical Nephrology 2005; 63: 381-4.

3. Khare CP. Encyclopedia of Indian Medicinal Plants 1982; 419-21.

4. Gil RR, Lin L, Cordell GA, Kumar M, et al. Anacardoside from the seeds of Semecarpus anacardium. Phytochemistry 1995; 39: 405-7.

5. Warren GV, Korbet SM, Schwartz MM, Lewis EJ. Minimal change glomerulopathy associated with non-steroidal antiinflammatory drugs. American Journal of Kidney Diseases 1989; 13: 127-30.

\section{H M N J Herath ${ }^{1}$, A W M Wazil ${ }^{1}$, N V I Ratnatunga ${ }^{2}$, A S Badurdeen ${ }^{1}$, K G A D Weerakoon ${ }^{3}$}

${ }^{1}$ Nephrology and Transplant Unit, Teaching Hospital, Kandy, ${ }^{2}$ Department of Pathology, Faculty of Medicine, University of Peradeniya, and ${ }^{3}$ Department of Parasitology, Faculty of Medicine and Allied Sciences, Rajarata University of Sri Lanka, Sri Lanka.

Correspondence: HMNJH, e-mail: <nalakajeewa@gmail.com>. Received 5 August and revised version accepted 11 November 2011. Competing interests: none declared. 